\title{
La (re)producción de las élites en tiempos de democratización del sistema universitario. Análisis conceptual a partir de las experiencias latinoamericanas ${ }^{*}$
}

\author{
María Luisa Quaresma** \\ Cristóbal Villalobos Dintrans ${ }^{* * *}$ \\ Recibido: 03 de febrero del 2018 \\ Enviado a pares evaluadores: 07 de febrero del 2018 \\ Aprobado por Comité editorial: 30 de mayo del 2018 \\ https://doi.org/10.22395/csye.v7n13a3
}

\section{RESUMEN}

El artículo desarrolla un análisis teórico-conceptual sobre el rol del sistema universitario en los procesos de movilidad y reproducción de la élite en el contexto de la democratización escolar. Se presenta una revisión de los principales debates sobre el concepto de élite y se analiza la relación entre (re)producción de la élite y universidad/carrera en países desarrollados y América Latina, con énfasis en dos países cuyos sistemas universitarios se caracterizan por sus altos niveles de democratización escolar y segregación interna: Chile y Brasil. Así, se propone una reflexión que muestra la importancia de la confluencia entre universidad y carrera en la configuración de los procesos de movilidad y reproducción social, entendiendo la configuración de la élite como un proceso dinámico y adaptativo.

Palabras clave: élite; educación superior; América Latina, reproducción social.

Este artículo resulta de un proyecto de investigación en curso titulado "Las élites académicas universitarias en Chile: un estudio sobre los perfiles, vivencias y percepciones del éxito que tiene el alumnado y el rol de las instituciones universitarias de élite en los procesos de reproducción y movilidad social", financiado por Conicyt (proyecto Fondecyt Regular número 1170371), en el cual la autora y el coautor de este artículo participan como investigadora responsable y coinvestigador, respectivamente.

** Doctora en Sociología de la Faculdade de Letras da Universidade do Porto (Portugal) y licenciada en Sociología de misma universidad. Profesora de la Universidad Autónoma de Chile, Facultad de Ciencias Sociales y Humanidades, e investigadora en el Instituto de Estudios Sociales y Humanísticos de la referida universidad. Correo electrónico: quaresma.ml@gmail.com

*** Doctor en Ciencias Sociales de la Universidad de Chile, magíster en Economía Aplicada a las Políticas Públicas de la Universidad Alberto Hurtado y la Georgetown University. Trabajador social y sociólogo de la Pontificia Universidad Católica de Chile. Investigador asociado del Centro de Estudios de Políticas y Prácticas en Educación (Ceppe-UC) de la Pontificia Universidad Católica de Chile. Correo electrónico: clvillal@uc.cl 


\section{Elites' (Re)production in the University System's Democratization Times. Conceptual Analysis based on Latin American Experiences}

\section{ABSTRACT}

The article develops a theoretical and conceptual analysis on the role of the university system in elite's mobility and reproduction processes in the context of educational democratization. A review of the main debates on the elite concept is presented, as well as an analysis on the relationship between elite's (re)production and university / career studied in developed countries and Latin America. Emphasis is made on two countries with university systems characterized by high levels of educational democratization and internal segregation: Chile and Brazil. Thus, a reflection is proposed that shows the importance of the confluence between university and career in the configuration of mobility processes and social reproduction, understanding the elite's configuration as a dynamic and adaptive process.

Keywords: elite; higher education; Latin America, social reproduction.

\section{A (re)produção das elites em tempos de democratização do sistema universitário. Análise conceitual a partir das experiências latino-americanas}

\section{RESUMO}

O artigo desenvolve uma análise teórico-conceitual sobre o papel do sistema universitário nos processos de mobilidade e reprodução da elite no contexto da democratização escolar. Apresenta-se uma revisão dos principais debates sobre o conceito de elite e analisa-se a relação entre (re)produção da elite e universidade/curso em países desenvolvidos e na América Latina, com ênfase em dois países cujos sistemas universitários se caracterizam por seus altos níveis de democratização escolar e segregação interna: o Chile e o Brasil. Assim, propõe-se uma reflexão que mostra a importância da confluência entre universidade e curso na configuração dos processos de mobilidade e reprodução social, entendendo a configuração da elite como um processo dinâmico e adaptativo.

Palavras-chave: elite; educação superior; América Latina, reprodução social. 


\section{Introducción}

Uno de los más importantes mecanismos de producción y reproducción de las élites es la educación superior. En la medida en que las sociedades logran mayores niveles de desarrollo, las certificaciones postsecundarias se hacen cada vez más necesarias para integrar cualquier grupo social privilegiado (Gérard y Wagner, 2015). Además, la expansión y democratización de la educación superior genera una lucha entre las clases sociales por oportunidades educacionales terciarias que les permitan conservar o aumentar sus ventajas posicionales.

Con el crecimiento de la matrícula postsecundaria, esta lucha se traslada del terreno cuantitativo (número de años de escolaridad) al cualitativo, por lo cual la disputa por los diplomas universitarios más selectivos y de mayor calidad cobra cada vez más fuerza. Por lo mismo, el aporte de estos diplomas no es parejo ni igualitario, sino que se desarrolla a través de una jerarquía de prestigio que privilegia a un "núcleo selecto" de carreras que garantizan altos ingresos, elevado reconocimiento social, contactos y redes sociales y seguridad económica futura (Brunner y Flisfich, 2014).

De esta forma, el valor simbólico de estos diplomas (y su valor de uso como recurso estratégico en los campos sociales y profesionales) dependen de la combinación de ciertas carreras en ciertas universidades, lo que permite perpetuar procesos de segmentación social y reproducción de diferencias a favor de los grupos más selectos de la sociedad. Así, mientras la carrera construye un prestigio social general y fija un propósito social al individuo, la universidad es una "marca de fábrica", que genera "una representación social compartida, provisoria y localizada, asociada a un nombre y resultante de evaluaciones más o menos poderosas y formalizadas" (Chauvin, 2013, p.3).

¿Cuál es entonces el rol de las universidades en la producción y reproducción de las élites contemporáneas? ¿De qué maneras utilizan las élites las instituciones de educación superior y las carreras como un espacio para su producción? El punto de referencia del presente artículo que busca responder a estas interrogantes es la realidad latinoamericana, con énfasis en los casos chileno y brasileño. De esta forma, se pretende precisar, a través de un análisis teóricoreflexivo, el uso que hacen las élites del espacio universitario como una forma de promover mecanismos de producción y reproducción social. Se postula que, en general, las élites construyen, preservan y validan reductos de excelencia y prestigio en determinadas carreras y ciertas universidades para garantizar la reproducción social de su grupo.

Se propone, de esta manera, entretejer un análisis conceptual que combine dos campos académicos relativamente diferenciados. Por una parte, un análisis 
sociológicamente fundamentado sobre los procesos de movilidad y reproducción social y el rol del sistema educativo en estos, para aportar así al debate sobre la histórica relación entre educación y estructura social. El complemento estriba en una reflexión que, desde la sociología de la educación, permita develar las características del sistema de educación superior y su configuración contemporánea, lo cual contribuye al debate sobre el mérito, la justicia y la igualdad de oportunidades en este espacio.

A diferencia de otros estudios, este análisis se desarrolla en base a tres premisas. En primer término -y a diferencia de un amplio espectro de estudios que buscan conocer y analizar quiénes son las actuales élites-, se hace una reflexión sociológica sobre los procesos de construcción de las futuras élites en el contexto universitario, lo que implica reconocer la noción de élite como un proceso anclado temporalmente en la historia de los sujetos, más que como una categoría instituida.

En segundo término, e íntimamente relacionado con lo anterior, se pretende revelar (y develar) el rol activo de las instituciones de educación superior en el proceso de producción y reproducción de las élites. Así, y a diferencia de la mayoría del conocimiento académico producido en el continente, se entiende la educación superior como un factor crítico (y no un simple proceso de continuidad "natural" entre la educación primaria y el trabajo) para la configuración de las élites.

Finalmente, se establece que este rol activo de la educación superior lo ejercen dos fuerzas: i) las universidades; y ii) las carreras. Como se indicó, se parte de la idea de que en esta combinación virtuosa entre universidades y carreras es que las élites construyen y configuran sus privilegios, lo que contrasta con los análisis existentes hasta la fecha, casi puramente concentrados en la figura de las universidades.

El objetivo es aportar al análisis conceptual contemporáneo sobre las élites y su relación con las universidades. Para ello, el artículo se divide en seis apartados. El apartado que sigue a esta introducción analiza el concepto de élite, haciendo énfasis en los significados contemporáneos de la misma. El tercer apartado describe las investigaciones y estudios de referencia sobre las élites en América Latina, se da cuenta de sus principales limitaciones y aportes. Los apartados cuarto y quinto argumentan conceptualmente sobre la relación entre élite y universidad, para dar evidencia de las jerarquías de prestigio que privilegian a un grupo reducido de establecimientos y de carreras, lo cual garantiza -en tiempos de democratización escolar- la (re)producción social de las clases dominantes en los países desarrollados, pero también en dos países latinoamericanos, tomados como ejemplos paradigmáticos de lo que ocurre en - 68 
América Latina: Chile y Brasil. Con base en todo lo anterior, el último apartado desarrolla una serie de conclusiones y reflexiones finales a manera de síntesis del aporte que el artículo ofrece para el análisis de la movilidad social y el desarrollo de los sistemas educativos.

\section{Élite: el concepto y las nuevas reconfiguraciones de sus significados}

Hablar de élite es hablar de un concepto polisémico (Moya, 2013) de difícil definición (Ball, 2015) y, en cierta medida, envuelto en controversia, una vez que o "[...] surge como connotación positiva, elogiosa, realzando calidades de individuos o grupos, o tiene una connotación negativa, crítica, responsabilizando personas o segmentos de la sociedad por las injusticias y desigualdades sociales" (Bottomore, 1965, p. 6). Se trata por tanto, de un concepto en disputa y continua revisión.

La discusión sobre el significado y conformación de las élites lo introdujeron Pareto (1916) y Mosca (1939) a las Ciencias Sociales, dos teóricos clásicos de la escuela italiana que desarrollaron los conceptos de élite gobernante y clase política respectivamente, para designar y dar cuenta de las minorías dominantes que en todas las sociedades logran someter a las mayorías. En la sociología norteamericana, el concepto lo popularizó Charles Wright-Mills (1957), quien desarrolló la idea de "élite del poder", la cual incorpora tres subélites responsables del destino de las mayorías: los dirigentes de empresas, los líderes políticos y los jefes militares. Los estudios de los sociólogos franceses Bourdieu y Passeron (1964; 1974) sobre el habitus, los capitales (económicos, pero también culturales, sociales y simbólicos) y la distinción también son aportes basales para la comprensión de estos grupos dominantes y de sus mecanismos de formación, reproducción y dominación.

Contemporáneamente, la discusión sobre las élites ha ganado renovada visibilidad social y sociológica (Ball, 2015), principal, aunque no exclusivamente, debido a la agudización de las diferencias sociales y económicas que el capitalismo transnacional y financiero genera entre un selecto grupo de individuos y una inmensa mayoría de ciudadanos (Piketty, 2014). Así, por ejemplo, Savage et al. (2013), definieron recientemente a la élite como el grupo social del país que posee, en todas las dimensiones, más ventajas y más privilegios, los cuales se traducen en la posesión de los más altos niveles de todas las formas de capitales (en el sentido bourdesiano del término). Al ser poseedora del más notable poder económico, cultural y social del país, la élite está, en general, constituida por un reducido y exclusivo grupo que se distingue por la sub-representación de las minorías étnicas y por la sobre-representación de egresados de las universidades con mayor reputación del país (Savage et al., 2013). 
En contraste Khan (2012a) prefiere no hablar de una sola élite, sino de élites (en plural), a las cuales define como aquellos grupos que se caracterizan por poseer un vasto y "desproporcionado acceso a o control de un recurso social" (Khan, 2012a, p. 362), con una condición particular: tener un "valor transferible" a otros espacios de la vida social. Así, y en función de la naturaleza de ese recurso, este sociólogo distingue entre élites económicas, políticas, sociales, culturales o de conocimiento.

Otra característica de las élites contemporáneas sería su alto nivel de diferenciación con respecto a la mayoría de los individuos. En esta línea, Khan (2012b) indica que las élites actuales son más ricas que las élites del pasado por su elevada participación en el mundo financiero. Paradójicamente, también se señala que las élites en el nuevo siglo son más inclusivas y receptivas frente a los valores meritocráticos, lo cual supone un nuevo rasgo identitario: haber pasado de ser "[...] una "clase" a una colección de individuos: -los mejores y los más talentosos" (Khan, 2012b, p. 480). De esta manera, para Khan las actuales élites no funcionarían necesariamente bajo una lógica de reciprocidad o apoyo, sino bajo la noción de competencia que impone el esfuerzo meritocrático (Young, 1958). Así, el esfuerzo y el talento individual serían los ingredientes que servirían para legitimar el privilegio, lo que permitiría que este fuera, además, percibido como aceptable porque sería considerado "justo" (Dubet, 2000).

Un rasgo igualmente diferenciador de las élites actuales consistiría en el dominio de un conjunto de habilidades "blandas" que les garantizarían ventajas significativas, especialmente en el campo profesional, en el cual se les valora mucho en la actualidad (Brown et al., 2016). Como observan Darchy-Koechlin et al. (2015, p. 144), las élites no poseerían únicamente "virtudes académicas" y altos niveles de conocimiento cognitivo y cultural, también dominarían valiosas aptitudes no-cognitivas, como la capacidad comunicativa y argumentativa, la auto-confianza, el sentido de responsabilidad, o la capacidad de liderazgo. La educación en colegios que privilegian la formación integral del individuo, como es el caso de los colegios de élite (Moya y Hernández, 2014), o la inserción en contextos familiares con elevados recursos económicos, culturales y relacionales potencian el desarrollo de estas habilidades blandas, fundamentales para la construcción de trayectorias profesionales, sociales y personales exitosas.

Finalmente, es importante entender el efecto del escenario de la globalización en el debate sobre las élites, que promueve la aplicación de conceptos clásicos a las nuevas realidades. En este sentido, no es de extrañar que durante los últimos años surgieran conceptos nuevos, como el de élite transnacional (Robinson, 2012) para designar a los grupos poderosos que mantienen contacto y redes internacionales; el de élite de la esfera económica para designar, por ejemplo, a los CEO 
(Chief Executive Officer) de las mayores empresas (Ellersgaard, Larsen y Munk, 2013); o la noción de "dominante dominantes" (MacLean, Harvey y Chia, 2010, p. 342 ), aplicada a aquellos sujetos que poseen una súper-concentración de poder, como es el caso de los directores Top 100 de las mayores empresas inglesas y francesas. En síntesis, y aunque es claro que existen diferencias con las élites estudiadas por los clásicos, las élites contemporáneas preservan con aquellas un rasgo en común que parece estar en el núcleo mismo de su definición: son potentes "motores de desigualdad social" (Khan, 2012a, p. 362).

\section{Las élites en América Latina. Un estado del arte}

En general, el tema de las élites ha sido poco trabajado en América Latina (Waldman, 2007). Esto es paradójico, considerando que es el continente más desigual del globo (Oxfam, 2015) y que, en estos contextos, los estudios sobre los grupos dominantes tienen el potencial de ayudar al entendimiento de los mecanismos que fundamentan las desigualdades sociales (Luci y Gessaghi, 2016).

En términos de investigaciones comparadas que abarquen el conjunto de la región, la producción académica sobre las élites es bastante limitada. Entre los estudios más relevantes sobre las élites sudamericanas está el clásico Élites y desarrollo en América Latina de Lipset y Solari (1967), que analiza el papel de las distintas minorías que controlan el poder, como es el caso de las élites políticas, religiosas, culturales, obreras, campesinas, pero también de la élite militar, que ha jugado un papel determinante en las dictaduras de la región y en la historia política del continente. Más recientemente, aun cuando focalizado solamente en las élites políticas, se encuentra el estudio de Sáez (2006) que parte de la premisa de que "los políticos importan", problematiza la contribución para la democracia que han realizado estos grupos, a partir del análisis de entrevistas a legisladores de 17 países sudamericanos. Estas se compendian en una importante obra colectiva que permite una comprensión del rol de la política desde sus propios actores. Desde una mirada más ecléctica, el libro de Birle et al. (2007), compila un conjunto de artículos sobre temas tan diversos como las élites criollas, las élites intelectuales-artísticas, los indígenas bolivianos y el campo de poder, el ascenso de los tecnócratas en México y Chile o la cooperación de los grupos dominantes para el desarrollo de sus países; entrega de esta manera, información sobre la configuración de distintos grupos de poder presentes en la región.

A diferencia de la información comparada, la investigación focalizada en élites de países particulares de la región es más abundante. En Colombia, el escenario del conflicto armado encausó el enfoque analítico hacia las élites y su relación con la emergencia de la lucha armada. Así, mientras Posada (2009) analizó las dinámicas de poder y de usurpación de tierras de las élites terratenientes, frecuentemente en articulación con los grupos de guerrilla 
y paramilitares, Romero (2000) se centró en las élites regionales y Mauceri (2001) relacionó la configuración fragmentada de las élites colombianas con la estrategia de abdicación-privatización adoptada por el Estado para combatir los movimientos armados.

En Argentina, el tema también ganó protagonismo en las últimas décadas. En este contexto, resaltan los estudios históricos que muestran las continuidades y rupturas en la construcción de la élite. Allí destacan: la contribución de Losada (2009) sobre las diferentes fracciones de la élite del país entre el período colonial y el peronismo, las investigaciones de De Imaz (1964) sobre los grupos dirigentes que entre 1936 y 1961 dominaban en Argentina, y los estudios de Hora (2003) sobre las poderosas clases propietarias rurales entre 1860 y 1945. Complementariamente, otras investigaciones han estudiado a los grupos dominantes actuales, Luci (2014) trabajó la dimensión cosmopolita de las élites actuales, representadas por los managers de las grandes empresas, para quienes la internacionalización de las carreras profesionales funciona como un mecanismo de distinción y jerarquización. Luci y Gessaghi (2016) se enfocaron en las familias tradicionales y las élites empresariales del país y reflexionaron sobre el rol de las redes de solidaridad y del trabajo de individuación meritocrática en la construcción, conservación y justificación de las posiciones de privilegio. Asimismo, Heredia (2011) y Castellani (2016) centraron sus planteamientos, respectivamente, en los ricos estructurales y los nuevos ricos de la capital, y en la élite económica argentina de la década del noventa. También es importante considerar los estudios sobre el rol de la educación en la formación de las clases dominantes, desarrollados por Gessaghi (2010), Ziegler (2017), Tiramonti y Ziegler (2008), y Méndez (2013).

La creación del Observatório de Elites Politicas e Sociais do Brasil es un hito significativo para esta área de estudios en Brasil. La creación de esta instancia va en consonancia con la revitalización de esta temática diagnosticada por Perissinotto y Codato (2008). En este país, los estudios pioneros del sociólogo Fernando Henrique Cardoso (1964; 1967) y de Bresser-Pereira (1974) sobre los empresarios brasileños tuvieron continuidad con los trabajos recientes de Costa et al. (2012) y de Pohlmann y Valarini (2013), que también se focalizaron en este tipo de élites. Asimismo, y de igual forma a lo ocurrido en Argentina, en Brasil se ha desarrollado una línea de investigación sobre la educación de las élites y de la relación entre sistema escolar y campo educativo en la cual destacan los trabajos de Nogueira (2002), Nogueira y Aguiar (2008), Almeida (2015; 2009) y Brandão (2007).

En Chile, la indiferencia de las ciencias sociales frente al tema de las élites la señalaron Joignant y Guell, autores de Tecnócratas y Mandarines (2011), una - 72 
de las obras más importantes sobre el tema hasta la fecha. Este libro compila un conjunto de contribuciones sobre la élite nacional, enfatiza en la élite gobernante, la tecnocracia y los procesos valóricos y educativos que están en el origen de la reproducción social de las élites, entrega así una panorámica general de dichas clases dominantes en Chile. Un tópico especialmente relevado en el país ha sido los mecanismos y formas de cierre social que aplican los grupos dominantes.

El estudio de Aguilar (2011) aborda las distintas dimensiones que adquieren las fronteras de la élite, mostrando así la combinación de factores materiales y simbólicos que se utilizan en el proceso de cierre social. En esta misma línea, el estudio de Stabili (2003) describió el habitus de la élite tradicional chilena, a través de testimonios femeninos que revelaron la importancia de dimensiones como el sentido de hidalguía o la fidelidad a las tradiciones. Complementariamente, los estudios de Thumala (2007), Hunneeus (2013) o Bowen (2015) han centrado su atención en las élites económicas, ya sea problematizando el ethos religioso de los ejecutivos de las grandes empresas, las "estrategias matrimoniales" (Bourdieu, 1989) utilizadas en la reproducción social de estas élites o las representaciones valórico-morales de los pobres compartidas por las élites económicas religiosas. Al igual que en otros países, allí los estudios sobre élites políticas también han estado presentes (Espinoza, 2010; Moya, 2013).

\section{La universidad y la formación de las élites. Diferenciación y (re)producción social}

Un tema central en el estudio de las élites (especialmente en las sociedades contemporáneas) es el rol de las instituciones escolares en su proceso de producción y reproducción (Moya y Hernández, 2014; Van Zanten, Ball y Darchy-Koechlin, 2015; Ziegler, 2017). Este proceso se desarrolla a lo largo de toda la trayectoria escolar del estudiante e inicia con una elección cuidadosa de la primera escuela (Van Zanten, 2015). De esta forma, la educación primaria y secundaria de élite busca, a través de rigurosos procesos de selección, elevadas mensualidades y clientelas con elevados poderes económicos, preservar el habitus de clase, crear capital social y generar privilegios posicionales dentro y fuera del campo educativo (Koh y Kenway, 2016; Bathmaker et al., 2016).

En la educación superior, la élite busca mantener y profundizar sus privilegios a través de mecanismos de distinción y diferenciación. De esta manera, los sistemas de educación superior se van produciendo y configurando. En las sociedades en las cuales se desarrollaron durante el siglo XX procesos de universalización de la educación secundaria, y democratización y expansión de la educación superior, la lucha entre las clases sociales por oportunidades educacionales terciarias, que les permitan conservar o aumentar sus ventajas posicionales, se traslada del terreno cuantitativo al terreno cualitativo. Como mencionamos 
anteriormente, en este escenario, las élites construyen, preservan y validan reductos de excelencia y prestigio en determinadas carreras y universidades, que les permitan garantizar que la reproducción social de su grupo se mantenga, a pesar de la expansión de la educación superior. En síntesis, es la combinación de ciertas carreras en ciertas universidades la que permite perpetuar procesos de segmentación social y reproducción de diferencias a favor de los grupos más selectos de la sociedad, lo que ha sido extensamente demostrado en diversos países desarrollados.

Así, por ejemplo, en un país como Francia, la dualidad valorativa de vías universitarias está representada, por un lado, por las Grandes Écoles estudiadas por Bourdieu (1989) y por otro, por las universidades de masas que acogen los públicos populares. Aunque se hayan adoptado políticas en el sentido de la apertura social de estas instituciones durante los últimos 20 años, distintas investigaciones (Van Zanten, 2010; Darchy-Koechlin et al., 2015) han señalado que, en general, estas siguen siendo un reducto de la juventud dominante, como resulta claro del perfil social de los alumnos de las "classes préparatoires" que dan acceso a sus selectivos concursos de admisión (Darmon, 2013). De esta manera, no extraña que dichas escuelas se interpreten como la "promesa seductora de un futuro profesional próspero" (Draelants, 2010, p. 351) catalizando, de esta manera, el ideal contemporáneo de la élite. Dicha apreciación no parece estar alejada de la realidad, toda vez que se ha comprobado que las Grandes Écoles son la vía real para acceder a las altas esferas científicas, políticas y administrativas de Francia y a los altos cargos dirigentes de las grandes empresas, que en el 2006 reclutaban cerca de 50\% de sus dirigentes de estos espacios (Massol, Vallée y Koch, 2010).

El caso de Francia, aunque sea paradigmático de la jerarquización de vías universitarias, no es el único. También en el Reino Unido "[...] el mero hecho de asistir a las universidades no garantiza el acceso a las futuras élites: el tipo de institución también importa" (Croxford y Raffe, 2015, p. 1626). Así, tal como observan MacMillan, Tyler y Vignoles (2015), gran parte del acceso a las ocupaciones de elevado status lo determina la universidad en la que se estudió. De esta manera, las más prestigiosas universidades del Russell Group -en el cual se encuentran Cambridge y Oxford, entre otras-, funcionan como un verdadero vivero de las élites británicas (Bathmaker et al., 2016; Jones, 2014), garantizando a sus egresados probabilidades tres veces mayores a las de las nuevas universidades para que ocupen cargos de élite en el mercado laboral, en áreas tan diversas como derecho, economía, periodismo o política (Jackson, 2009).

Las universidades del Russell Group junto con la University of London Colleges se consideran una especie de súper-élite de las universidades, que, según Wakeling y Savage (2015), confirman el rol facilitador de las universidades de 
renombre para el acceso a posiciones socialmente ventajosas, especialmente en términos de recursos económicos. Un hecho que confirma este privilegio es que los ex alumnos de Oxford, por ejemplo, tienen un ingreso familiar 50\% superior al de los colegas de la universidad ubicada en el puesto 23 de los rankings nacionales.

En cuanto a los Estados Unidos, la evidencia apunta en direcciones bastante similares. El estudio de Karabel (2005) identificó las tres instituciones universitarias (Harvard, Yale y Princeton) en las cuales está sobrerrepresentada la élite estadounidense. Aunque actualmente se muestren más abiertas a la heterogeneidad racial, étnica y religiosa, en el marco de sucesivas re-definiciones del concepto de mérito, estas universidades siguen siendo reductos privilegiados de elegidos, lo cual evidencia una "masiva sub-representación de pobres y de estudiantes de la clase obrera" (Karabel, 2005, p. 554), tal como ocurre en otras universidades selectivas.

Para Espenshade y Radford (2011), esta sub-representación no es producto de los mecanismos (más o menos explícitos de admisión), sino también del proceso de autoselección/autosegregación que las familias y jóvenes desarrollan al momento de ingreso al sistema superior, en el cual aspectos económicos, culturales y sociales se entremezclan y codefinen. De esta manera, serían las élites las que construirían y reconstruirían la propia noción de mérito en el sistema educativo norteamericano, como una forma para reflejar de mejor manera los valores y capacidades de su propio grupo, estableciendo el "pedigree" necesario para un futuro laboral exitoso (Rivera, 2016).

Aunque muy informativa y consistente, este conjunto de evidencias tiende a concentrarse en entender el rol de las instituciones de educación superior en los procesos de producción de la posición social en países desarrollados, con altos niveles de renta y en los cuales la educación superior se encuentra masificada desde hace décadas. En contraste, la siguiente sección presenta la evidencia disponible hasta la fecha sobre el proceso de producción y reproducción de la élite en dos países con procesos de masificación universitaria recientes y con los más elevados niveles de desigualdad educativa en América Latina: Brasil y Chile.

\section{Educación superior y reproducción social en América Latina. Los casos de Chile y Brasil}

Al igual que en otros contextos geográficos, también en América Latina el sistema de enseñanza superior es una vía privilegiada de producción y reproducción de las élites y un universo que actualmente se caracteriza por "su heterogeneidad institucional, su fuerte diversificación y diferenciación", rasgos desarrollados en el marco del cambio "de un modelo universitario de élite bastante homogéneo 
y cerrado a un sistema con características más populares, de índole masiva" (Olivier, 2012) que condujo al fuerte aumento del sector privado.

En este contexto, Brasil y Chile son dos casos interesantes. En primer lugar, ambos países son casos paradigmáticos de privatización universitaria, con más del $50 \%$ de la matrícula en el sector privado (Olivier, 2012), lo cual ha provocado crecientes tensiones en ambos contextos. En segundo lugar, Chile y Brasil representan casos de expansión universitaria explosiva, que ha alcanzado niveles de masificación similares a los de los países industrializados (Brunner y Villalobos, 2014) y que por lo mismo, temáticas como el financiamiento y la calidad de la enseñanza se han puesto en la mirada pública en la última década. Finalmente, tanto Brasil como Chile han propulsado en esta última década importantes reformas a sus sistemas de admisión universitaria, con el objetivo de integrar a poblaciones históricamente excluidas de este nivel educativo (Villalobos et al., 2017). Esto ha dado pie a discusiones profusas sobre el talento, el mérito y la justicia educativa. Por todas estas razones, estos países pueden entenderse como ejemplos centrales del proceso de masificación, privatización, segmentación social y diferenciación académica propios de las sociedades latinoamericanas, siendo, por lo tanto, casos ejemplares para el estudio de la relación entre élites y educación superior.

Ahora, con respecto a las investigaciones sobre las élites, es interesante notar que los estudios disponibles presentan similitudes y diferencias en ambos países. En el caso de Brasil, Almeida (2015) observa que la formación de enclaves sociales y académicos empieza desde el inicio de la trayectoria escolar. Así, los hijos de las familias privilegiadas reciben educación en prestigiosos y exclusivos colegios del sector privado, algunos con un perfil internacional que atiende el deseo de internacionalización común en las élites brasileñas, como observan Windle y Nogueira (2015). Por otro lado, los descendientes de las clases populares tienen como única alternativa la red de educación pública, que ha perdido calidad de forma constante desde la década de los setenta (Almeida, 2015).

Así, al momento de ingresar a la educación superior, los "herederos" (Bourdieu y Passeron, 1964) se concentran en las más selectivas carreras de un núcleo reducido de prestigiosas universidades, que incluye esencialmente estabelecimientos públicos y gratuitos, pero también algunos particulares y pagados, como ciertas universidades católicas y algunas pocas escuelas profesionales tuteladas por fundaciones no lucrativas. Las prestigiosas universidades de São Paulo y Campinas, por ejemplo, acogían en el 2000 solamente el 22,2\% y el 30,7\%, respectivamente, de alumnos provenientes de colegios públicos. La baja presencia de jóvenes pertenecientes a familias desfavorecidas económica y culturalmente es aún más notoria en las carreras más selectivas de cada una de 
estas universidades: Medicina (5,3\% y 7,3\%), Ingeniería Mecánica (6,0\% e 4,8\%) y Economía (6,8\% y 4,3\%). De esta manera, queda en evidencia que la reproducción de la élite se manifiesta por la combinación de procesos de autoselección, trayectorias diferenciadas y procesos de selección institucional, prácticamente indiferenciables entre sí.

A pesar de la gradual implementación de políticas que buscan la apertura de la universidad pública a los grupos populares, esencialmente a través del mecanismo de las cuotas (Villalobos et al., 2017), las élites brasileñas no solamente mantienen sus antiguos enclaves universitarios, sino que han generado estrategias que les han permitido expandir su radio de acción, conquistando así nuevos nichos. Como señala un estudio sobre la expansión del sector público universitario y la apertura de nuevas extensiones en las Universidades Federal y Estatal de São Paulo (Almeida 2015), mientras los campus más antiguos de estas universidades (ubicados en los mejores barrios de la ciudad y con la oferta de las carreras de más prestigio) siguen concentrando a las élites, los nuevos campus (construidos en los barrios pobres y con la oferta de las carreras de menos prestigio) concentran principalmente a los jóvenes de las clases trabajadoras, lo cual conlleva procesos de jerarquización que incluyen como factor central la gestión y diferenciación del espacio social.

Otro estudio de Almeida y Ernica (2015) sobre la inclusión y segmentación social en la enseñanza superior pública de São Paulo también concluye que la relativa inclusión de las clases populares coexiste con el sostenimiento de la tradicional segmentación de la educación superior brasileña, lo que permite que las carreras más prestigiosas se mantengan relativamente cerradas a los grupos sociales menos favorecidos. Así, en la Universidad Federal de São Paulo, por ejemplo, el 64\% de los estudiantes que ingresaron en el 2012 provenían de padres sin formación superior o de hogares de bajo nivel socioeconómico y aún así, se evidenciaba una fuerte concentración de estudiantes de alto status socioeconómico y simbólico en carreras como Administración o Medicina -que en el 2012 acogían respetivamente el 80\% y el 77,9\% de jóvenes de los estratos favorecidos-, y que por el contrario, otro grupo de carreras concentra a los estudiantes de los estratos más pobres, como ocurre con Ciencias Sociales, Servicio Social, Filosofía o Pedagogía -estas acogían, respectivamente, el 64,6\%, el 63,9\%, el 58,5\% y el $48 \%$ de jóvenes social y económicamente más desfavorecidos-.

En el caso del sector privado, la elección de las élites se concentra en un grupo selecto de universidades, generalmente ubicadas en barrios "nobles". Algunas de estas universidades se reconocen por su selectividad académica y por la calidad de su formación en áreas como Economía o Administración Pública, otras se reconocen por la selectividad social garantizada por el filtro 
de elevadas mensualidades, lo cual posibilita el acceso a carreras tradicionales, como Ingeniería o Economía o aún a carreras menos convencionales, como Moda o Diseño Gráfico. Según Pholmann y Valarini (2013), cerca de la mitad de los top managers de las mayores empresas brasileñas concluyeron su carrera universitaria en universidades particulares de renombre y sólida reputación académica.

En su modelo de clasificación de las universidades privadas brasileñas, García (2005) identifica como instituciones de élite a: la Fundação Getúlio Vargas (FGV), espacio de referencia en la formación de las élites económicas (Codato et al 2016) y parlamentarias (Simoni et al., 2016); la Faculdade de Economia e Finanças (Ibmec); y la Escola Superior de Propaganda e Marketing (ESPM). En el "cuadrante de los sueños" del sector privado, García (2005) incluye a la Pontificia Universidad Católica (PUC), también destacada en la formación de los líderes económicos (Codato et al. 2016) y políticos (Simoni et al., 2016).

Por otra parte, Chile dispone en su sistema nacional de educación superior "de un núcleo institucional que proporciona la plataforma para la tarea formativa de las élites" (Brunner, 2012, p. 122). Como observa Brunner, si antes de la reforma universitaria de 1967 y 1968 las ocho universidades del país eran un reducto exclusivo de la élite tradicional chilena que aseguraba la "propia reproducción como grupo" (Aguilar, 2011, p. 227), con la masificación de la educación superior esa labor pasó a ser garantizada por una minoría de prestigiosas universidades que hoy coexisten con instituciones educativas que preparan las masas y que son mayoría (Fleet y Guzmán-Concha, 2016). Así, en el contexto del notorio incremento de la población matriculada la matrícula, que pasó de 25.000 estudiantes en 1960 a 1.130 .0000 en 2012 (Brunner 2014), Chile se encuentra frente a la multiplicación y la diferenciación de las organizaciones que proveen educación superior (Brunner y Villalobos, 2014). Uno de los rasgos diferenciadores más inmediato es el nivel de selectividad, con importantes implicaciones sobre las escalas de prestigio y la reputación social de estas instituciones. Los retornos a la educación terciaria presentan gran heterogeneidad en función de la universidad a la que se asiste (Urzúa, 2012; Espinoza, 2013) -que impacta en el tipo de cargo al que se accede (Neira, 2004) y en los niveles de empleabilidad (Meller, 2010)-, pero también en función de la carrera que se elige, de igual forma, allí se presentan niveles diferenciados de renta mensual (Espinoza, 2013).

De esta forma, y confirmando que la reputación se transfiere de la institución a los individuos que asisten a ella (Chauvin, 2013), los alumnos de las universidades de élite tienen amplias ventajas económicas en comparación con los estudiantes de las universidades de masas (Fleet y Guzmán-Concha, 2016), lo que también ocurre entre los estudiantes de las universidades del Consejo de Rectores de las Universidades de Chile (Cruch) en comparación con los de las nuevas universidades privadas (Meller, 2010), se combinan por tanto criterios 
históricos y de calidad en la reproducción social de las élites. De la misma manera, los jóvenes que eligen carreras como Ingeniería, Medicina o Derecho presentan numerosas ventajas sociales y profesionales con respecto a los que eligen Pedagogías o Artes (Mineduc, 2010), se demuestra así el alto grado de diferenciación y segregación del sistema educativo universitario mercantilizado consolidado en los últimos 25 años (Villalobos, 2016).

Respecto a las universidades y carreras de élite, estas se caracterizan por poseer altos niveles de calidad y prestigio, garantizados mediante un proceso de selección riguroso pero que "no reduce de manera automática el campo de reclutamiento a los herederos, quienes, de cualquier manera, en su mayoría, tienen asegurado el acceso a instituciones de este tipo, sino que sirve además como filtro meritocrático para reclutar nuevos hombres y mujeres que alimenten la circulación (micro, interna) de aquellos grupos" (Brunner, 2012, p. 122). Entre estas universidades, se encuentran especialmente dos: la Universidad de Chile (UCH) y la Pontificia Universidad Católica de Chile (PUC), espacios de formación de la élite gobernante tecnocrática chilena (Brunner, 2012). Ambas se encuentran posicionadas en los primeros puestos de los rankings universitarios de América Latina, son las instituciones que imparten las más prestigiosas y selectivas carreras del país, esencialmente Derecho, Ingeniería y Economía y Negocios.

Estas carreras y universidades producen la élite del futuro, son las instituciones y estudios de los cuales egresan los altos ejecutivos del país, pero también las figuras más relevantes de la vida política. Joignant (2011) analizó las trayectorias de un grupo de veinte technopols (actores que fusionan altos niveles de capitales técnicos y políticos) antes y después de haber cumplido el rol de ministros y subsecretarios en los primeros veinte años de transición democrática (1990-2010) e identificó los dos rasgos más determinantes de este grupo: la universidad y la carrera. Así, la gran mayoría de los technopols cursaron su pregrado en las dos universidades mencionadas y egresaron, en su gran mayoría, de las carreras de Derecho (ocho) y Economía (siete).

Por otra parte, Zimmerman (2015) constató que los estudiantes de Ingeniería o Derecho de la UCH o la PUC presentan mayor probabilidad de ocupar posiciones de liderazgo en las grandes empresas del país. Los resultados demuestran la importancia del background social para el acceso a los cargos de élite empresarial (medida a través del colegio asistido). Lo anterior confirma la amplia ventaja de los jóvenes de las clases privilegiadas y revela los límites de la movilidad social ascendente. Así, Zimmerman (2015) concluye que el 5\% de los egresados de colegios particulares de élite constituye el $39 \%$ de los top managers, mientras el $4 \%$ de las escuelas públicas de élite apenas da cuenta del $5 \%$ de estos altos ejecutivos. 


\section{Conclusiones}

El estado actual de la investigación sobre las clases dominantes y sus procesos de producción y reproducción social en América Latina es dinámico y creciente. En aquellos países en los cuales los sistemas educativos son altamente desiguales y estratificados (como en América Latina, especialmente en Chile y Brasil), la discusión sobre el rol del espacio educativo en estos procesos ha cobrado renovado interés durante los últimos años. Sin embargo, la mayor parte de la literatura existente tiende a concentrarse en: i) visualizar las estrategias y mecanismos de reproducción de las élites en la educación primaria o secundaria; ii) estudiar las actuales élites económicas, políticas y sociales, los estudios secundarios y/o terciarios solo se incorporan como una variable explicativa a posteriori.

A contrapelo, la presente reflexión visualiza el concepto de élite y sus procesos de producción y reproducción como un proceso cambiante temporalmente, se hace hincapié en el rol de la formación terciaria en el proceso de configuración de la élite. De esta manera, se buscó desarrollar una reflexión teórica que diera cuenta de la importancia del punto de encuentro entre universidad y carrera para la configuración de los procesos de movilidad o reproducción social. Se evidencia de esta manera que el proceso de formación es dinámico en el tiempo. En la figura 1 se puede apreciar una representación gráfica simplificada del mismo.

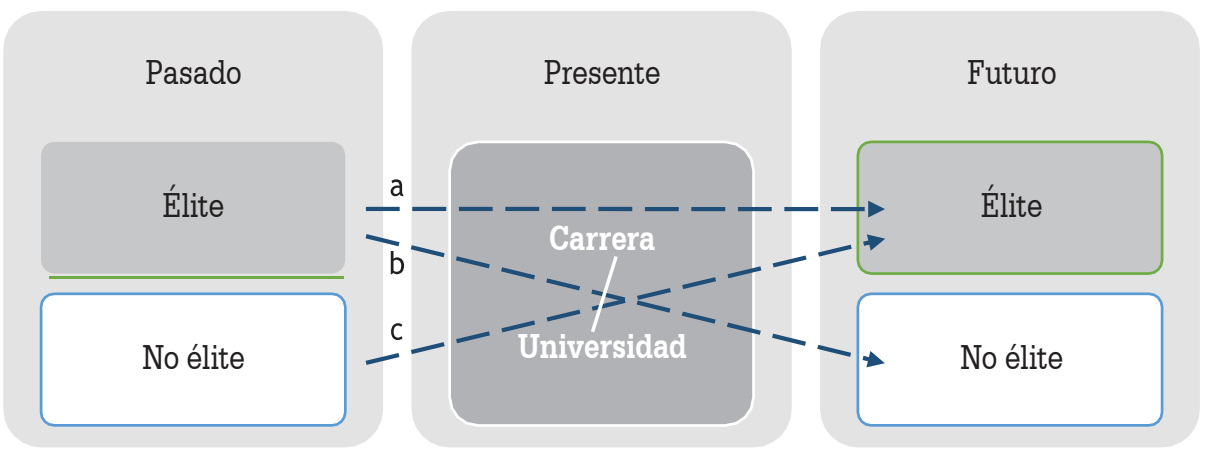

Nota: En letras las diferentes trayectorias: (a) reproducción; (b) desvanecimiento; y (c) generación.

Figura 1. Mapa de trayectorias universitarias de élite Fuente: elaboración propia

Como se puede observar, existen tres momentos o pilares temporales de producción y reproducción de la élite organizados en torno al rol de la educación terciaria: i) la herencia (representada por el pasado del sujeto), que corresponde al proceso de socialización familiar, socioeconómico y educativo anterior a la universidad; ii) la pertenencia (da cuenta del presente del individuo), que corresponde al proceso de producción y reproducción realizado en el espacio terciario 
en el cual resultan centrales el nivel de estatus y privilegio de las carreras y universidades y; iii) la referencia (proyectada en el futuro), que da cuenta del proceso de formación y/o consolidación de la élite, sea en términos de su capital económico, político o intelectual y que constituye la "imagen de marca" de la institución también para estudiantes y familias.

De esta manera, se pretende relevar el rol activo de la formación post-secundaria en la producción y reproducción social. Se identificaron tres dinámicas o trayectorias de producción y/o reproducción de las élites. En primer término (a), están los tradicionales procesos de reproducción de las élites, que surgen mayoritariamente en relación con la conservación del nivel socioeconómico y que se garantizan con e estudio de carreras como Ingeniería Civil, Ingeniería Comercial o Derecho en prestigiosas universidades. En estos casos, las universidades y carreras actúan como un locus de formación que consolida la posición social recibida "en herencia", esto genera trayectorias de continuidad élite-élite.

En segundo lugar (b), se deben considerar los procesos de movilidad ascendente garantizados (o por lo menos facilitados) por la asistencia a una carrera/universidad, lo que compondría procesos de generación de élites. En este caso, carreras como Medicina o determinadas ramas de la Ingeniería en universidades selectivas, pero no necesariamente prestigiosas, pueden jugar un papel fundamental puesto que actúan como "resorte" para la consolidación de nuevos grupos sociales emergentes que se consolidan a través de la adquisición de ciertos capitales (en general, económicos), posteriormente utilizados o intercambiados por otras formas de capital (políticos, culturales, intelectuales). En estos casos, el rol de la carrera/universidad es contribuir a la reconfiguración del espacio social de élite, de manera que le abra la puerta a los que, gracias a los procesos educativos, logran romper las barreras sociales.

Finalmente, este análisis requiere también una reflexión sobre los procesos de movilidad "descendente" (c), no en el sentido que la sociología tradicionalmente le atribuye, sino en el sentido de lo que podría llamarse como desvanecimiento de la élite. Así, estos casos no se caracterizan necesariamente por la convencional caída en la escalera social, ya que, como documenta la literatura, las élites poseedoras de conciencia de clase auto-preservan el capital adquirido en herencia, cerrando sus fronteras a quienes no han adquirido, por vía familiar, su ethos. Por el contrario, se trata de procesos que se caracterizan por la asistencia a carreras en universidades (generalmente de prestigio) que no garantizan, per se, los más altos niveles de ingreso o reconocimiento social, político o intelectual. Esta elección se basa, esencialmente, en motivaciones personales de cariz expresivo ("la vocación") o aún, en estrategias funcionales de división del trabajo familiar/social. 
Ejemplos de este tipo de trayectorias podrían ser carreras poco prestigiosas socialmente (como las Pedagogías o el Arte) en entornos altamente prestigiosos (es decir, universidades selectivas y/o privadas). En estos casos, el espacio terciario actúa como un espacio de desarrollo personal, cuyo retorno esperable es la "profesionalización de la vocación" y no un retorno de naturaleza económica o social. Menos expresivas numéricamente que las anteriores, estas elecciones configuran también una marca identitaria de los herederos, cuya pertenencia social altamente protegida les permite compartir aspiraciones que obedecen más al principio del placer que al de la realidad (Bourdieu y Passeron, 1964).

Aunque este sea un abordaje preliminar y todavía de naturaleza teórica, la reflexión sobre el rol de las universidades y carreras en los procesos de producción y reproducción de la élite permite expandir las fronteras del entendimiento sobre la relación entre universidad y procesos de reconfiguración social, dando cuenta de las instituciones de educación superior como espacios no solamente de formación, sino de educación y de socialización, y también como lugares centrales para la reproducción, generación o desvanecimiento de las élites, esto ofrece nuevos insumos para la investigación académica sobre clases sociales y sistema educativo.

\section{Referencias}

Aguilar, O. (2011). Dinero, educación y moral: el cierre social de la élite tradicional chilena. En: A. Joignant y P. Güell (Eds.), Notables, tecnócratas y mandarines. Elementos de Sociología de las élites en Chile (1990-2010), (pp. 185-202). Santiago: Ediciones Diego Portales.

Almeida, A. M. (2009). As escolas dos dirigentes paulistas. Ensino médio, vestibular, desigualdade social. Belo Horizonte: Argumentum Editora.

Almeida, A. M. (2015). The changing strategies of social closure in elite education in Brasil. En A. Van Zanten, S. Ball y B. Darchy-Koechlin (Eds.), Elites, privilege and excellence. The national and global redefinition of educational advantag (pp. 70-81). Nueva York: Routledge.

Almeida, A. M., y Ernica, M. (2015). Inclusão e segmentação social no Ensino Superior público no Estado de São Paulo (1990-2012). Educacao e Sociedade, 36(130), 63-83.

Ball, S. (2015). Elites, education and identity. An emerging research agenda. En A. Van Zanten, S. Ball y B. Darchy-Koechlin (Eds.), Elites, privilege and excellence. The national and global redefinition of educational advantag (pp. 233-240). Nueva York: Routledge.

Bathmaker, A. M., Ingram, N., Abrahams, J., Hoare, A., Waller, R., y Bradley, H. (2016). Higher Education, Social Class and Social Mobility. The Degree Generation. London: Palgrave Macmillan.

Birle, P., Hofmeister, W., Malhold, G., y Potthas, B. (2007). Elites en América Latina. Madrid-Frankfurt: Iberoamericana, Editorial Vervuert.

Bottomore, T. (1965). As elites e a sociedade. Rio de Janeiro: Zahar.

Bourdieu, P. (1989). La noblesse d'Etat. Grandes écoles et esprit de corps. Paris: Les Editions de Minuit.

- 82 
Bourdieu, P., y Passeron, J.C. (1964). Les héritiers. Paris: Les Editions de Minuit.

Bourdieu, P. y Passeron, J.C. (1974). A reprodução-elementos para uma teoria do sistema de ensino. Lisboa: Editorial Vega.

Bowen, S. (2015). Educar la moral del pobre: fronteras simbólicas y gobierno de los pobres por parte de la élite económica católica de Santiago de Chile. Pro-Posições, 26(2), 51-73.

Brandão, Z. (2007). A produção das elites escolares: escolas, famílias e cultura. Caderno CRH, 20(49), 15-22.

Bresser-Pereira, L. (1974). Empresários e administradores no Brasil. São Paulo: Brasiliense.

Brown, P., Power, S., Gerbrand, T., y Allouch, A. (2016). Credentials, talent and cultural capital: a comparative study of educational elites in England and France. British Journal of Sociology of Education, 37(2), 191-211.

Brunner, J. J. (2012). La lucha por la educación de las élites: campo y canales formativos. Revista UDP, 9, 119-143.

Brunner, J. J., y Flisfisch, Á. (2014). Los intelectuales y las instituciones de la cultura. Santiago: Ediciones Universidad Diego Portales.

Brunner, J. J., y Villalobos, C. (2014). Políticas de educación superior en Iberoamérica, 2009-2013. Santiago: Ediciones Universidad Diego Portales.

Cardoso, F. H. (1964). Empresário industrial e desenvolvimento econômico no Brasil. São Paulo: Difel.

Cardoso, F. H. (1967). Las élites empresariales en América Latina. En Seymour L. y Solaris, A. (eds.). Elites y desarrollo en América Latina. Buenos Aires: Paidós.

Castellani, A. (2016). Radiografía de la élite económica argentina. Estructura y organización en los años noventa. Buenos Aires: Unsam Edita.

Chauvin, P. M. (2013). La sociologie des réputations. Une définition et cinq questions. Communications, (93), 131-145.

Codato, A., Cavalieri, M., Perissinotto, R., y Dantas, E. (2016). Economic mainstream and power: a profile analysis of Central Bank directors during PSDB and PT governments in Brazil. Nova economia, 26(3), 687-720.

Costa, P., Roks, T., y Santos Filho, G. (2012). Recrutamento, valores e padrões de ação política da elite empresarial. Revista de Sociologia e Política, 20(43), 221-246.

Croxford, L. y Raffe, D. (2015). The iron law of hierarchy? Institutional differentiation in UK higher education. Studies in Higher Education, 40(9), 1625-1640.

Darchy-Koechlin, B., Draelants, H., y Tenret, E. (2015). National and international students' definition of merit in French Grandes Écoles. En A. Van Zanten, S. Ball y B. Darchy-Koechlin (Eds.). Elites, privilege and excellence. The national and global redefinition of educational advantag. Nueva York: Routledge.

Darmon, M. (2013). Classes préparatoires. La fabrique d'une jeunesse dominante. Paris: La Découverte.

De Ímaz, J. L. (1964). Los que mandan. Buenos Aires: Eudeba.

Draelants, H. (2010). Les effets d'attraction des grandes écoles. Excellence, prestige et rapport à l'institution. Sociologie, 3(1), 337-356. 
Dubet, F. (2000). L'égalité et le mérite dans l'école démocratique de masse. L’année Sociologique, 50(2), 383-408.

Ellersgaard, C., Larsen, A., y Munk, M. (2013). A Very Economic Elite - The Case of the Danish Top CEOs. Sociology, 47(6), 1051-1071.

Espenshade, T., y Radford, A. (2011). No Longer Separate, Not Yet Equal: Race and Class in Elite College Admission and Campus Life. Princeton, NJ: Princeton University Press.

Espinoza, Ó. (2013). Equidad e inclusividad en el sistema de educación superior en Chile. En Óscar Espinoza (Ed.), Equidad e Inclusividad en la educación superior en los países Andinos: Los casos de Bolivia, Chile, Colombia y Perú (pp. 93-133). Santiago de Chile: Ediciones Universidad UCINF.

Espinoza, Ó., y González, L. (2011). La crisis del sistema de educación superior chileno y el ocaso del modelo neoliberal. En Barómetro de Política y Equidad, Nuevos actores, nuevas banderas (pp. 94-133). Santiago: Fundación Equitas-Fundación Friedrich Ebert.

Espinoza, V. (2010). Redes de poder y sociabilidad en la élite política chilena. Los parlamentarios 19902005. Polis, Revista de la Universidad Bolivariana, 9(26), 251-286.

Fleet, N. y Guzmán-Concha, C. (2016). Mass higher education and the 2011 student movement in Chile: material and ideological implications. Bulletin of Latin American Research, Special Issue, 1-17.

García, M. (2005). Planejamento estratégico. Revista Ensino Superior, (77), 41-43.

Gérard, É., y Wagner, A. C. (2015). Introduction: Élites au Nord, élites au Sud: des savoirs en concurrence? Cahiers de la recherche sur l'éducation et les savoirs, (14), 7-24.

Gessaghi, V. (2010). Trayectorias educativas y clase alta. Etnografía de una relación (Disertación doctoral). Universidad de Buenos Aires, Argentina.

Heredia, M. (2011). Estructurales y nuevos ricos en Buenos Aires: primeras pistas sobre la reproducción y la recomposición de las clases altas. Estudios Sociológicos, 29(85), 61-97.

Hora, R. (2003). Los terratenientes de la pampa argentina. Una historia social y política 1860-1945. Buenos Aires: Siglo Veintiuno.

Huneeus, S. (2013). Estrategias de reproducción matrimonial de la élite económica en Chile. Revista de Sociología, (28), 67-82.

Jackson, M. (2009). Disadvantaged through discrimination the role of employers in social stratification. The British Journal of Sociology, 60(4), 669-692.

Joignant, A. (2011). Tecnócratas, technopols y dirigentes de partido: tipos de agentes y especies de capital en las élites gubernamentales de la Concertación (1990-2010). En A. Joignant y P. Güell (Eds.), Notables, tecnócratas y mandarines. Elementos de Sociología de las élites en Chile (1990-2010). Santiago: Ediciones Universidad Diego Portales.

Joignant, A., y Güell, P. (Eds.), Notables, tecnócratas y mandarines. Elementos de Sociología de las élites en Chile (1990-2010). Santiago: Ediciones Universidad Diego Portales.

Jones, O. (2014). The establishment: And how they get away with it. Harmondsworth: Allen Lane.

Karabel, J. (2005). The chosen: The hidden history of admission and exclusion at Harvard, Yale and Princeton. Boston: Houghton Mifflin.

Khan, S. (2012a). The Sociology of elites. Annual Review of Sociology, (38), 361-377.

- 84 
Khan, S. (2012b). Elite identities. Identities: Global Studies in Culture and Power, 19(4), 477-484.

Koh, A., y Kenway, J. (2016). Introduction: Reading the Dynamics of Educational Privilege through a spatial lens. En: A. Koh y J. Kenway (Eds.), Elite schools: Multiple Geographies of Privilege (pp. 1-15). Nueva York: Routledge.

Lipset, S. y Solari, A. (1967). Élites y desarrollo en América Latina. Buenos Aires: Editorial Paidós.

Losada, L. (2009). Historia de las élites en la Argentina: desde la conquista hasta el surgimiento del peronismo. Buenos Aires: Editorial Sudamericana.

Luci, F. (2014). La "internacional de los managers" cultura cosmopolita, movilidad internacional y éxito corporativo en las grandes empresas globales. A Contracorriente: Revista de Historia Social y Literatura en América Latina, 11(2), 166-194.

Luci, F., y Gessaghi, V. (2016). Familias tradicionales y élites empresarias en Argentina: individuación y solidaridad en la construcción y sostén de las posiciones de privilegio. Política, 54(1), 53-84.

MacLean, M., Harvey, C., y Chia, R. (2010). Dominant Corporate Agents and the Power Elite in France and Britain. Organization Studies, 31(3), 327-348.

MacMillan, L., Tyler, C., y Vignoles, A. (2015). Who gets the top jobs? The role of family background and networks in recent graduates' access to high-status professions. Journal of Social Policy, 44(3), 487-515.

Massol, J., Vallée, T., y Koch, T. (2010). Lés élites économiques sont-elles encore si différentes en France et en Allemagne?. Regards sur l'économie Allemande, (97), 5-14.

Mauceri, P. (2001). Estado, élites y contrainsurgencia: una comparación preliminar entre Colombia y Perú. Colombia Internacional, (52), 44-64.

Meller, P. (2010). Rol de la universidad en la determinación del ingreso de los profesionales. En P. Meller y B. Lara (Eds.), Carreras Universitarias. Rentabilidad, selectividad y discriminación (pp. 103-125). Santiago: Uqbar.

Méndez, A. (2013). El colegio. La formación de una élite meritocrática en el Nacional Buenos Aires. Buenos Aires: Sudamericana.

Mineduc (2010). Futuro Laboral 2009-2010. Profesionales y Técnicos en Chile: información fundamental. Santiago de Chile: Ministerio de Educación.

Mosca, G. (1939). The ruling class: Elementi di scienzi política. London: McGraw- Hill.

Moya, E. (2013). Entre la espada y la pared. Los discursos sobre la élite política en el contexto de la globalización. Un estudio de tres casos, Chile, Argentina y México. X Jornadas de Sociología. Facultad de Ciencias Sociales. Universidad de Buenos Aires, Buenos Aires.

Moya, E. y Hernández, J. (2014). El rol de los colegios de élite en la reproducción intergeneracional de la élite chilena. Revista Austral de Ciencias Sociales, (26), 59-82.

Neira, H. (2004). Educación universitaria en Chile: una visión panorámica centrada en los alumnos. Estudios Pedagógicos, (30), 123-133.

Nogueira, M. A. (2002). Estratégias de escolarização em famílias de empresários. En A. M. Almeida y M. A. Nogueira (Eds.). A escolarização das elites. Um panorama internacional da pesquisa. Petrópolis: Editorial Vozes.

Nogueira, M. A., y Aguiar, A. (2008). La formation des élites et l'internationalisation des études: peuton parler d'une "bonne volonté internationale"?, Education et sociétés, 1(21), 105-119. 
Olivier, G. (2012). Reto de la educación superior privada en América latina: entre la expansión y la resistencia. Recuperado de https://ideas.revues.org/382

Oxfam (2015). Privilégios que negam direitos. Desigualdade extrema e sequestro da democracia na américa latina e o caribe. Recuperado de https://www.oxfam.org/sites/www.oxfam.org/files/file_attachments/ cr-privileges-deny-rights-inequality-lac-300915-summ-pt_0.pdf

Pareto, V. (1916). Trattato di Sociologia Generale. Vol. II. Firenze: Barbera.

Piketty, T. (2014). El capital en el Siglo XXI. México D.F.: Fondo de Cultura Económica.

Perissinotto, R., y Codato, A. (2008). Apresentação: por um retorno à Sociologia das Elites. Revista de Sociologia e Política, 16(30), 7-15.

Pohlmann, M. y Valarini, E. (2013). Elite econômica no Brasil: discussões acerca da internacionalização da carreira de executivos brasileiros. Revista de Sociologia Politítica, 21(47), 39-53.

Posada, A. (2009). Guerreros y campesinos. El despojo de la tierra en Colombia. Norma: Bogotá.

Programa de Naciones Unidas para el Desarrollo (PNUD) (2015). Desarrollo Humano en Chile. Los tiempos de la politización. Santiago: PNUD Chile.

Rivera, L. (2016). Pedigree: How Elite Students Get Elite Jobs. Princeton, NJ: Princeton University Press.

Robinson, W. (2012). Global Capitalism Theory and the Emergence of Transnational Elites. Critical Sociology, 38(3), 349-363.

Romero, M. (2000). Changing Identities and Contested Settings: Regional Elites and the Paramilitaries in Colombia. International Journal of Politics, Culture and Society, 14(1), 51-69.

Sáez, M. (2006). Políticos y política en América Latina. Madrid: Fundación Carolina/Siglo XXI.

Savage, M., Devine, F., Cunningham, N., Taylor, M., Li, Y., Hjellbrekke, J., Miles, A. (2013). A New of Social Class? Findings from the BBC's Great Class Survey Experiment. Sociology, 47(2), 219-250.

Simoni Junior, S., Dardaque, R., y Mingardi, L. (2016). A elite parlamentar brasileira de 1995 a 2010: até que ponto vai a popularização da classe política? Colombia Internacional, (87), 109-143.

Stabili, M. R. (2003). El sentimiento aristocrático. Élites chilenas frente al espejo (1860-1960). Santiago: Editorial Andrés Bello y Centro de Investigaciones Diego Barros Arana.

Thumala, M. A. (2007). Riqueza y Piedad. El catolicismo de la élite económica chilena. Santiago: Editorial Debate.

Tiramonti, G. y Ziegler, S. (2008). La educación de las élites. Aspiraciones, estrategias y oportunidades. Buenos Aires: Paidós.

Urzúa, S. (2012). La rentabilidad de la educación superior en Chile: revisión de las bases de 30 años de políticas públicas. Estudios Públicos, (125), 1-52.

Van Zanten, A. (2010). L'ouverture social des grandes écoles: diversification des élites ou renouveau des politiques publiques d'éducation? Sociétés Contemporaines, (78), 69-96.

Van Zanten, A. (2015). A family affair reproducing elite positions and preserving the ideals of meritocratic competition and youth autonomy. En A. Van Zanten, S. Ball y B. Darchy-Koechlin (Eds.), Elites, privilege and excellence. The national and global redefinition of educational advantage (pp. 29-42). Nueva York: Routledge

- 86 
Van Zanten, A, Ball, S., y Darchy-Koechlin, B. (2015). Elites, privilege and excellence: the national and global redefinition of educational advantage. Nueva York: Routledge

Villalobos, C. (2016). El campo educativo en Chile post-dictadura (1990-2013). Continuidad y ruptura en la implementación del neoliberalismo en educación. En A. Pinol (Ed.), Democracia versus neoliberalismo. 25 años de neoliberalismo en Chile (pp. 159-178). Santiago: Fundación Rosa Luxemburgo-ICAL.

Villalobos, C., Treviño, E., Wyman, I., y Scheele, J. (2017). Social justice debate and college access in Latin America: merit or need? The role of educational institutions and states in broadening access to higher education in the region. Archivos Analíticos de Políticas Educativas, 25(73), 1-31.

Wakeling, P., y Savage, M. (2015). Elite universities, elite schooling and reproduction in Britain. En A. Van Zanten, S. Ball y B. Darchy-Koechlin (Eds.), Elites, privilege and excellence. The national and global redefinition of educational advantage (pp. 169-184). Nueva York: Routledge.

Waldmann, P. (2007). Algunas observaciones y reflexiones críticas sobre el concepto de élite. En P. Birley y W. Hofmeister (Eds.), Élites en América Latina (pp. 9-30). Berlín, Vervuert: Bibliotheca IberoAmericana.

Windle, J., y Nogueira, M. A. (2015). The role of internationalization in the schooling of Brazilian elites: distinctions between two class fractions. British journal of education, 36 (1), 174-192

Wright-Mills, C. (1957). La élite del poder. México D.F.: Fondo de Cultura Económica.

Young, M. (1958). The Rise of the Meritocracy, 1870-2033. London: Thames y Hudson.

Ziegler, S. (2017). Personalization and competition in elite schools in Buenos Aires: school strategies for the production and legitimization of dominant groups. Journal of education and work, 30(2), 145-155.

Zimmerman, S. (2015). Making Top Managers: The Role of Elite Universities and Elite Peers. Recuperado de: www.drive.google.com/file/d/0B3o_Y3MSsVJ3TXh1aW12cjF6TXc/view 